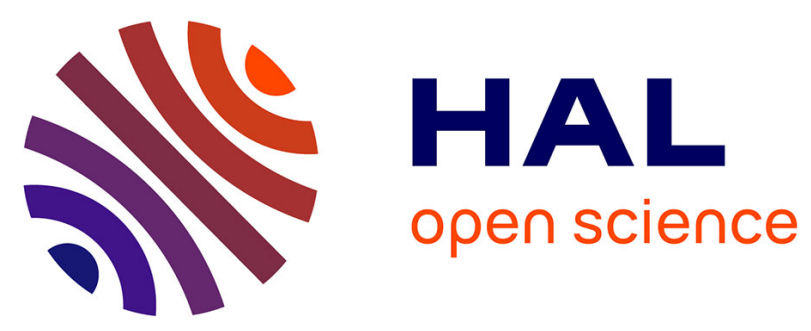

\title{
Le mémoire professionnel comme mise en scène d'une problématisation
}

\author{
Annette Gonnin-Bolo, Denis Lemaître
}

\section{To cite this version:}

Annette Gonnin-Bolo, Denis Lemaître. Le mémoire professionnel comme mise en scène d'une problématisation . Recherche et formation, 2005, n 48, pp. 31-45. hal-00526172

HAL Id: hal-00526172

https://hal-ensta-bretagne.archives-ouvertes.fr/hal-00526172

Submitted on 18 May 2016

HAL is a multi-disciplinary open access archive for the deposit and dissemination of scientific research documents, whether they are published or not. The documents may come from teaching and research institutions in France or abroad, or from public or private research centers.
L'archive ouverte pluridisciplinaire HAL, est destinée au dépôt et à la diffusion de documents scientifiques de niveau recherche, publiés ou non, émanant des établissements d'enseignement et de recherche français ou étrangers, des laboratoires publics ou privés. 


\section{LE MÉMOIRE PROFESSIONNEL COMME MISE EN SCÈNE D'UNE PROBLÉMATISATION}

\section{Annette Gonnin-BOLO* et Denis LemaîTRE**}

Résumé

L'injonction à problématiser est devenue récurrente dans nombre de cursus de formation de haut niveau et le mémoire professionnel, soutenu en fin d'études, est censé être un lieu privilégié où cette capacité se manifeste et peut s'évaluer. Mais l'expérience montre que le dispositif ne va pas de soi. L'objectif de l'article est d'examiner en quoi le mémoire professionnel peut effectivement représenter le support d'une problématisation en actes. Après avoir explicité différentes modalités caractéristiques du processus de problématisation, nous avons analysé des mémoires issus de trois contextes professionnels différents (école d'ingénieurs, IUFM et école d'orthophonie). Nous avons tenté, d'une part, de caractériser ce que le sujet (re)présente de la problématisation à travers les procédés d'écriture, d'autre part, de dégager le sens et les limites de l'exercice.

Abstract

The ability to problematize has become a recurrent requirement in many high level training courses, and the professional dissertation defended at the end of studies is supposed to be a privileged place where this ability is expressed and can be assessed. However, experience shows that this system is not so obvious. The aim of this paper is to study how the professional dissertation can actually represent the medium of the act of problematization. After analysing dissertations coming from three different professional contexts (a College of Engineering, a Teacher Training College and a School for Speech

* - Annette Gonnin-Bolo, université de Nantes; CREN.

** - Denis Lemaître, ENSIETA, Brest; CREN. 
Resumen

Therapists), we have tried, on the one hand, to define what the subject represents in terms of problematization through the writing devices, on the other hand, to bring out the meaning and limits of this task.

\section{Resumen}

Zusammenfassung In zahlreichen Studiengängen fortgeschrittener Ausbildung wird die Problematisierung häufig verlangt und die Examsarbeit (die am Ende des Studiums verteidigt wird) ist eigentlich der günstige Ort, wo sich diese Tätigkeit äußert und evaluiert werden kann. Die Erfahrung zeigt aber, dass dieses Verfahren nicht selbstverständlich ist. Dieser Artikel setzt sich als Ziel zu prüfen, inwiefern die Examsarbeit tatsächlich der Träger einer Problematisierung sein kann. Nachdem wir verschiedene für die Problematisierung typische Modalitäten erklärt hatten, haben wir Examsarbeiten analysiert, die aus drei verschiedenen beruflichen Milieus stammten (Ingenieurschule, pädagogische Hochschule, Logopädieschule). Wir haben versucht, einerseits zu charakterisieren, was das Thema durch die Ausdrucksmittel von der Problematisierung darstellt und andererseits den Sinn und die Grenzen der Übung herauszuarbeiten. 
Nombre de formations professionnelles à cursus long (médecin, ingénieur, architecte, enseignant, avocat, etc.) ont dans leurs objectifs de développer une compétence particulière à la problématisation, dans le champ d'expertise visé: il peut $s^{\prime}$ agir aussi bien de l'établissement d'un diagnostic, de l'élaboration d'une stratégie $d^{\prime}$ intervention ou de remédiation, de la conception de dispositifs techniques pour répondre à des besoins nouveaux. Cette capacité à problématiser s'évalue souvent en fin de cursus, au travers d'une mise en situation (stage) restituée par un mémoire, qui est censé faire la preuve que le futur professionnel maîtrise la complexité du contexte qui donne sens aux actions. Mais il s'agit d'un exercice difficile pour les étudiants, qui n'en perçoivent pas toujours bien le sens et qui se heurtent à la difficulté de maîtriser la rhétorique du genre.

Le mémoire se présente comme un écrit tout à la fois personnel, professionnel et institutionnel (Gonnin-Bolo, 2002). C'est au travers de ces trois dimensions que le stagiaire est invité à construire une problématique. Ce qui dans le stage existe par des gestes, des paroles, des réflexions en actes, se doit en effet d'être reproduit a posteriori sous la forme écrite du mémoire professionnel, par l'usage d'artifices dont la maîtrise est elle-même un obstacle. S'il y a effectivement problématisation dans la mise en situation professionnelle (ce qui n'est pas forcément acquis), ou dans la réflexion concourant à la rédaction du mémoire, il y a encore un pas pour que ce processus de problématisation soit effectivement reproduit et mis en scène à la hauteur des attentes du formateur-évaluateur.

La réflexion proposée ici s'oriente donc vers le questionnement du mémoire comme lieu effectif de problématisation d'une situation professionnelle. Que peut-on attendre en effet de ce genre textuel académique pour mettre en scène une compétence à problématiser? Sous quelles formes évoque-t-on, dans les mémoires, les mises en problème vécues? Peut-on, par la lecture des mémoires professionnels, repérer des traits caractéristiques de problématisation? L'enjeu important qui se dessine derrière ce questionnement est celui du statut à donner à l'exercice du mémoire dans la validation d'une formation professionnelle.

\section{L'ANALYSE DE MÉMOIRES EN FORMATION PROFESSIONNELLE}

Faisant donc l'hypothèse que la mise en forme d'une problématisation, évaluée comme trace d'une compétence professionnelle par l'évaluateur du mémoire, est elle-même très problématique, nous nous sommes intéressés aux mémoires produits par les étudiants issus de trois filières professionnelles différentes (ingénieurs, enseignants, et orthophonistes) pour examiner les formes d'expression que peut prendre cette mise en problème. 
Les exemples retenus sont:

- trois mémoires en école d'ingénieurs (1): en fin de troisième année, ce mémoire rend compte des capacités que l'étudiant a pu démontrer lors de son stage en entreprise pour résoudre un problème $d^{\prime}$ 'ingénieur; il doit manifester un niveau scientifique et technique, une réflexion et une prise de recul sur un problème industriel, tout en valorisant les résultats et l'expérience développée;

- un mémoire fait par les enseignants en formation en IUFM; ce type de mémoire est sans doute "le plus jeune» introduit dans une démarche de formation puisqu'avant les IUFM (1991) il n'existait pas; il exige une démarche qui s'apparente à une démarche de recherche articulant pratique et théorie;

- un mémoire de fin d'études en orthophonie; il se situe dans une tradition des études médicales et paramédicales et se veut un outil de production de savoirs pour la profession; il doit être "scientifique » et fait encore souvent appel à une méthode expérimentale rigoureuse, mais cette démarche, si elle est scientifique, $n^{\prime}$ implique pas obligatoirement une problématisation des questions posées.

Le choix de ces formations différentes permet de prendre en compte des formalisations particulières aux contextes concernés, sans négliger le fait que, même si des textes de cadrage existent, les mémoires peuvent différer aussi à l'intérieur même $d^{\prime}$ un secteur professionnel et au sein d'une même institution. Cette problématisation ne porte pas sur les mêmes objets en fonction des attentes exprimées dans chacune des formations professionnelles considérées (enseignant, orthophoniste, ingénieur). L'observation des mémoires nous montre ainsi que les problèmes qu'analysent leurs auteurs ne sont pas tous du même registre. Dans certains cas l'approche est très technique, dans d'autres elle est plus personnelle, dans certains cas plus réflexive, dans d'autres plus descriptive. Les représentations du problème diffèrent (Fabre, 1999).

D'un point de vue méthodologique, notre approche repose sur l'idée que le processus de problématisation est mis en scène par un ensemble de procédés rhétoriques (mise en valeur d'obstacles, de dépassements, etc.) et par le mouvement même des mémoires censé représenter des cheminements intellectuels. La mise en forme par l'écriture révèle différentes modalités du problématique, qui tiennent tant à la nature du métier visé, aux usages institutionnels de l'établissement de formation, à la culture du lieu visité en stage, qu'aux aspirations du stagiaire et à la manière dont il interprète les injonctions de ses encadrants. Ces différentes modalités d'appréhension des problèmes tournent autour du degré de subjectivation qu'y mettent les étudiants, selon qu'ils insistent sur leur réflexion et leur action personnelles, ou qu'ils en restent à une évocation distanciée des faits. En s'inspirant des catégories de modalités que Bernard Pottier (1992) établit dans le domaine de la linguistique, on peut

1 - Trois mémoires d'ingénieurs contre un de chaque pour les enseignants et les orthophonistes, car le volume plus réduit et le caractère très technique des mémoires $d^{\prime}$ 'ingénieurs demandaientt plusieurs exemples pour un examen pertinent de la question. 
distinguer: ce qui relève du constat («le monde est ainsi »), ce qui relève de la pensée ("je pense que»), ce qui relève de l'action («je fais, je peux, je dois faire»), ce qui relève de l'évaluation ("c'est mieux»). Ces quatre catégories de modalités linguistiques nous permettent $d^{\prime}$ identifier le niveau auquel les auteurs de mémoires situent le problématique. On peut ainsi définir des modalités du problématique: ontique (le constat de ce qui est), épistémique (savoir produit par le sujet), pragmatique (action intentionnée), axiologique (évaluation, jugement de valeur).

\section{EN ÉCOLE D'INGÉNIEURS}

Les trois mémoires d'élèves-ingénieurs étudiés ici (portant sur trois problèmes industriels différents) ont tendance à situer le problématique au niveau du constat de ce qui existe dans le monde. Dans les introductions (début de première partie) la situation instable identifiée (opposition, contradiction, paradoxe...) est celle de l'entreprise ou du secteur industriel considéré, ex.: "Durant les dernières années, la quantité de matériaux composites utilisée dans l'aéronautique a augmenté de manière significative. Néanmoins, la fabrication de pièces composites se fait, de nos jours encore, essentiellement de manière manuelle. » Dans la suite des mémoires on entre directement dans la description technique de l'existant et des procédures de traitement du problème. Le problème, tel que présenté, s'inscrit dans une objectivité générale qui n'est pas justifiée ou interrogée. Il n'y a pas de mise en scène du sujet épistémique ("je pense que », "j'en déduis que »). C'est la science et la réalité technique qui s'imposent aux stagiaires, comme des données non questionnables. On demeure ainsi dans une modalité ontique (les choses qui existent) correspondant à une approche réaliste du monde.

Ainsi, dans ces mémoires, passe-t-on très rapidement de l'obstacle technique présenté de manière détachée à la solution qui s'impose, elle aussi, de manière "naturelle», le travail du stagiaire étant présenté comme la validation de cette solution, dans une logique applicative. La "présentation du projet d'études » suivant, portant sur la construction de cuves de navires, est à ce titre assez représentative: "Les armateurs, clients de $\mathrm{N}$, exigent dorénavant des durées de vie de 40 ans dans des conditions de mer extrêmes pour les navires. La finalité du projet est donc très claire, elle doit permettre de valider [...] un assemblage mécano-soudé du système $X$ capable de résister 40 ans en Atlantique Nord [...]. L'étude devra faire apparaître l'existence ou non de zones probables de rupture [...] et proposer des solutions de renforcement $[\ldots] »$.

Juste après cette présentation du problème, l'auteur du mémoire présente la "démarche adoptée et [les] moyens mis en œuvre», sous la forme d'une énumération des contenus du mémoire: "Ce rapport résume la démarche suivante: 
- appréhension des efforts subis par la structure ainsi que des conditions aux limites;

- modélisation de l'ensemble;

$-[\ldots]$

- solutions envisagées».

Les modalisations employées par l'auteur sont particulièrement révélatrices d'une volonté de présenter le problème et son traitement comme des choses non questionnables: "est très claire », "doit permettre », "devra donc faire apparaître ». Telles que présentées, la raison d'être du projet et de l'approche suivie par le stagiaire ne sont pas le fait de son travail d'ingénieur (construction et formalisation du problème à partir des difficultés et des besoins exprimés par un client, en l'occurrence l'entreprise $N$ qui fabrique les cuves), mais sont imposées par le contexte, et sont donc aux yeux du stagiaire - incontestables. Même si elle s'est très probablement manifestée au cours des six mois de stage, il n'y a pas au sein du mémoire de mise en scène d'une quelconque distance critique vis-à-vis de la question posée et des injonctions de l'entreprise. La " démarche " présentée dans l'organisation du mémoire n'est pas montrée non plus comme le fruit d'une réflexion critique mais comme un déroulement qui s'impose, et dont la logique n'est pas mise en valeur - bien au contraire - par le procédé d'énumération retenu pour la rédaction. Ainsi évite-t-on soigneusement de mettre en scène une quelconque réflexivité.

De manière générale, le mouvement adopté dans chacun des trois mémoires ne représente pas une véritable démarche de problématisation. Tout juste y a-t-il au début énoncé d'une difficulté, et à la fin énoncé de solutions possibles ou de la solution retenue - sans que celles-ci soient discutées. C'est la campagne de calculs ou de programmation (processus technique décrit dans le corps du mémoire) qui aboutit à des données quantitatives, elles-mêmes validées ou invalidées par rapport au cahier des charges initial. Dans ce contexte les solutions s'imposent $d^{\prime}$ elles-mêmes, sans autre réflexion sur la faisabilité ou les enjeux.

Cette posture est sans doute à expliquer par le rapport à la réalité qu'entretiennent ces élèves-ingénieurs, marqués par une forme de réductionnisme positiviste, ainsi que par la stratégie déployée par eux pour désamorcer les critiques des évaluateurs. "Ce n'est pas moi, c'est le monde qui impose cette démarche» semble vouloir dire le stagiaire, alors que le métier d'ingénieur, pour l'exercice duquel on évalue précisément ses capacités, exige au contraire de remettre en cause les évidences du monde et de problématiser les obstacles rencontrés, en vue de produire des innovations (Lemaître, 2003).

On observe fréquemment dans la rédaction de ces mémoires une volonté d'éviter la mise en scène du problématique: comment le problème est réputé exister dans le 
monde, comment je le fais mien, comment je le construis, comment je choisis d'orienter l'action, comment j'évalue les résultats obtenus... Les mémoires d'ingénieurs étudiés éludent ainsi la dimension cognitive du sujet. On ne peut affirmer qu'il n'y a pas travail de problématisation, mais dans les représentations du stagiaire ce travail $n^{\prime}$ est pas assimilé à de la science ou à de la maîtrise technique, telles qu'elles doivent être présentées dans un mémoire et évaluées.

\section{EN INSTITUT DE FORMATION DES MAÎTRES}

Le choix s'est porté sur un mémoire qui aborde un sujet assez "classique» dans les mémoires, à savoir la motivation des élèves et leurs difficultés à apprendre. Le titre Comment aider les élèves en difficulté scolaire à progresser? est une question professionnelle, qui appelle des réponses situées dans le domaine de l'action et suppose donc la modalité pragmatique ("qu'est-ce que je dois faire pour? »).

Le stagiaire commence par présenter l'établissement, les classes où il enseigne, et livre quelques données sur les élèves. Ces données se veulent des données du problème à traiter (âge, redoublement, choix de la section...) mais restent descriptives. À la lecture il n'est pas possible de déterminer le rôle que jouent ces données pour le sujet: s'agit-il de contraintes?

Puis le stagiaire pose le problème: parti dans son premier cours d'un schéma d'enseignement cours-exercices-contrôle, il prend conscience que les élèves ne comprennent pas et "se relâchent". En analysant ce premier cours il cherche à identifier les éléments pertinents pour pouvoir comprendre ce qui se passe effectivement. Il commence alors à construire le problème. Cette étape se décompose en différentes phases.

\section{Les élèves sont retenus comme données premières du problème}

L'enseignant décide de construire un questionnaire pour les élèves: "Je me suis interrogé sur les causes possibles d'un manque de motivation " nous informe-t-il. On voit là que, réduisant le champ des possibles, il ne retient dans l'analyse qu'une dimension qui est celle de la motivation des élèves, notion «prête à penser » s'il en est dans le milieu enseignant - mais rappelons qu'il s'agit d'un enseignant novice.

Manque de motivation générale, manque de motivation pour les mathématiques, sont présentées comme indépendantes du sujet qui écrit le mémoire, selon une modalité ontique (les choses sont ainsi). 
La démarche du questionnaire est un premier élément dans une "construction tâtonnante » du problème, qui paraît flou. Dans la mesure où une seule donnée, même complexe (la motivation des élèves) est retenue, nous pouvons sans doute, à la suite de M. Fabre, qualifier la démarche de "pensée plate ", car il n'y a pas déploiement des deux axes de la problématisation (les conditions et les données).

Les réponses que donnent les élèves au questionnaire montrent en fait un intérêt pour les mathématiques (pour leur projet professionnel et leur propre instruction), mais ce sont leurs "difficultés » qui reviennent le plus souvent. Le stagiaire abandonne alors la motivation comme élément du problème et avance "un découragement face aux retards accumulés».

\section{Apparaît alors une rupture dans la démarche, ef il commence à analyser ses pratiques}

L'enseignant devient la donnée principale du problème: il analyse la construction de ses séquences $d^{\prime}$ apprentissage et remet ainsi en cause ses propres pratiques. Il fait un détour par certaines lectures (arrive alors une première référence bibliographique). $S^{\prime}$ appropriant ainsi des savoirs construits à l'extérieur (dans le champ des sciences de l'éducation) et les combinant à ses propres savoirs sur son activité, il adopte sur la question de l'apprentissage des élèves une posture épistémique. Apparaît un dédoublement, selon deux dimensions, des données et des conditions. Le contexte problématique se construit, l'enseignant entame une réflexion sur son action.

L'enseignant passe alors de l'analyse de la situation à l'action sur la situation: il présente alors une série de séquences pédagogiques et analyse ce qu'elles produisent. Mais dans sa présentation les séquences s'enchaînent de façon additive, car il procède par tâtonnements. On se situe dans le bricolage pédagogique. Les outils de compréhension destinés à l'analyse relèvent de différents registres: il s'agit tout autant de la surcharge cognitive qui décourage les élèves, de la sensibilité du stagiaire et de ses affects ("i'ai été agréablement surprise de voir les élèves s'impliquer »), que d'une certaine doxa pédagogique ("les élèves restent actifs tout au long du travail quand ils perçoivent le sens»). Il est difficile de parler de "problématisation systématisée ", c'est-à-dire posée dès le départ avec un objectif, car même si l'on observe une complexification croissante des données prises en compte dans la construction du problème celles-ci restent "dans le désordre ", la compatibilité et la cohérence entre elles n'étant pas établie. Les données se superposent sans s'organiser, et les conditions du problème n'apparaissent que de façon morcelée. 


\section{Par un retour fécond sur l'acte d'apprendre, le stagiaire remet en cause sa pensée}

Il se rend compte à ce moment qu'il lui faut sans doute réfléchir plus largement et en amont des phénomènes. Ainsi revient-il sur l'ensemble des données pour poser les conditions de l'apprentissage: "J'ai pu alors déterminer les conditions qui me semblent indispensables à un nouvel apprentissage. " $\|$ procède à une évaluation diagnostique pour adapter ses stratégies de formation en fonction des compétences et des aptitudes des élèves, puis entre dans une démarche d'évaluation formative pour mieux repérer et accompagner leurs acquis et leurs difficultés. II s'agit de "faire en sorte que l'élève remarque ses erreurs mais aussi ses réussites" et d'apporter des remèdes.

Mais il poursuit dans la juxtaposition d'analyses et d'actions.

De nouvelles conditions s'ajoutent aux précédentes. Chaque action nouvelle est présentée avec des arguments scientifiques tirés de la littérature pédagogique, et $s^{\prime} a p$ puie sur des données factuelles. À chaque fois le problème est posé avec quelques données, puis se reconfigure sur des données et des conditions, mais il ne reste ni ouvert ni fermé. Sa construction demeure dans l'inachevé, comme une simple étape.

Au total, cet enseignant cherche à "résoudre» un problème mais il reste trop prisonnier de la nécessité de l'action pour prendre le temps nécessaire de le construire (il s'agit d'un enseignant novice, rappelons-le). Il procède par tentatives successives, entre pratiques mises en œuvre et analyses des résultats de ses pratiques, de façon fragmentée. Dans le cadre du mémoire, il met en scène, avec une certaine honnêteté, ses tâtonnements et ses réflexions, sans que le problème soit véritablement construit. À la suite de M. Guigue (1995), nous parlerons alors de "pensée relationnelle en émergence », et non de véritable problématisation.

\section{EN ÉCOLE D'ORTHOPHONIE}

Après l'examen d'une dizaine de mémoires d'orthophonie il est apparu que, beaucoup moins codés dans la forme que les mémoires IUFM, ils en étaient très différents. Dans ce type de formation le modèle du mémoire s'apparente davantage à celui d'une mini-recherche, pouvant aller jusqu'à des protocoles expérimentaux de type médical pour tester l'efficacité de techniques de diagnostic ou de rééducation. Un peu comme les élèves-ingénieurs les étudiants doivent produire des savoirs plutôt utiles à la "science», éclairant sur l'efficacité de techniques de soins ou de diagnostics, ou utiles à la profession en éclairant des aspects non étudiés des pratiques professionnelles et leur impact sur d'autres acteurs (familles, écoles...). Le sujet (l'orthophoniste qui rédige le mémoire) problématise une question qui lui reste extérieure, il ne fait pas lui-même partie du problème. 
Nous avons choisi d'étudier un mémoire qui porte sur le bégaiement d'enfants, car il nous paraît intéressant notamment quant à la traduction que se fait l'étudiant des attentes de l'institution: Le rôle de l'environnement familial dans la rééducation du jeune enfant bègue.

L'étudiant part du constat que le bégaiement est un trouble qui entraîne une grande souffrance chez les enfants. Les familles sont démunies face à ce trouble (données présentées selon une modalité ontique, c'est-à-dire comme appartenant au domaine de la connaissance générale). Mais au-delà de ces données de départ émerge la question "de la dynamique familiale qui règle les différentes interactions de ses membres: parent-enfant, parent-parent, enfant-enfant». II s'agit alors d'analyser les paramètres de la communication dans la famille susceptibles d'améliorer ou d'aggraver la parole de l'enfant bègue, puis d'évaluer ce que peut changer la prise en charge orthophonique et ce que les parents en retiennent et en comprennent. Le problème posé est donc à double dimension.

\section{L'éłudiant vérifie si le problème posé est légitime, avec comme référence les travaux scientifiques sur la question}

Dans une première partie il fait le point sur différentes théories du bégaiement. Dans cette démarche, les théories sont présentées de façon cumulative, et comme indépendantes. Sur certains points même elles peuvent apparaître comme contradictoires, ce qui amène l'étudiant à conclure "qu'à l'heure actuelle nous sommes encore très ignorants dans ce domaine». Et c'est au nom de la pratique qu'il retient l'une de ces théories pour bâtir sa problématique: "En matière de thérapie, ce modèle multifactoriel, prenant en compte les différents aspects du bégaiement, semble le plus efficace » (on sait ce qui marche, mais on ne sait pas vraiment pourquoi). D'un point de vue thématique, le rôle de l'environnement - et donc celui des parents - est l'une des données du problème sur laquelle va s'appuyer l'étudiant. II s'agit dès lors pour lui de définir des conditions qui vont permettre de remédier à ce trouble dans le développement de l'enfant.

\section{Une démarche qui se veut scientifique malgré la tentation de l'exhaustivité}

Comme il ne peut s'appuyer sur des données scientifiques sûres, il propose un questionnaire aux parents en posant le plus de questions possibles pour couvrir toutes les données susceptibles d'entrer dans la problématique (le questionnaire comprend cinquante deux questions). Pour autant le problème n'est pas construit: s'agit-il de mieux connaître ces enfants qui bégaient et la configuration familiale dans laquelle ils se trouvent? Dans ce cas la fonction des données est-elle descriptive ou explica- 
tive? Est-ce de mieux connaître les attitudes de la famille vis-à-vis de l'enfant? Là aussi la fonction des données (et leur analyse) peut être descriptive ou au contraire devenir des conditions d'explication de certaines évolutions, ce qui constituait l'un des problèmes posés au départ. La construction du questionnaire montre un élargissement flou du problème. L'étudiant pose plusieurs questions en même temps sans les organiser, et ne parvient pas à définir les données et les conditions de son problème. L'étudiant tire un portrait très vaste (et très intéressant) de ce que sont ces enfants, comment apparaît le bégaiement, comment réagissent les parents, l'entourage, comment cela se passe globalement dans la famille. Certaines données sont analysées en termes d'inférences, à travers le discours des parents: ce que les parents considèrent comme un facteur d'aggravation ou d'atténuation du bégaiement (moment de la journée, situation, émotions...). Mais cela reste des données extérieures à l'attitude propre des parents. L'étudiant fait aussi référence à des témoignages d'orthophonistes venus d'ailleurs pour insister sur l'importance du contexte de communication et de relation dans le bégaiement. Il semblerait que ce soit bien là le centre du problème traité mais la mise en scène du mémoire l'oblige à évoquer d'autres éléments qui ne seraient que des "accessoires superflus». La mise en relation des données (bégaiement) et des conditions (communication dans la famille), qui pourrait constituer une démarche de problématisation, aurait pu être mise en évidence sans le recours au questionnaire; rien en effet dans ce questionnaire (croisement ou réflexions des parents) ne permet d'aller plus loin. Son analyse est en quelque sorte une mise en scène "savante» pour affirmer ce que l'étudiant savait déjà et voulait justifier.

\section{Un recentrage sur le problème traité?}

L'étudiant revient au deuxième problème traité: le rôle de la rééducation dans l'évolution du comportement des parents face aux enfants.

Il tente alors de montrer par des tableaux croisés (en articulant les données et les conditions, il passe en deux dimensions) que la rééducation et les conseils donnés lors de la prise en change ont apporté un changement significatif des attitudes des parents face au bégaiement de l'enfant. Mais là encore il semble que le recueil et l'analyse chiffrée des données ne servent qu'à confirmer les hypothèses. Puis par un retour réflexif sur son cheminement «scientifique», l'étudiant montre les limites de cette confirmation Mais cette réflexion porte sur la méthode de vérification des hypothèses, non sur l'hypothèse elle-même.

I| reprend la construction du problème: il nuance la portée de l'implication des parents dans la thérapie en fonction de l'enfant ("chaque enfant étant singulier »). D'une problématisation qu'il veut générale au départ il passe ainsi à une problématisation fermée sur l'individualité (chaque couple parents/enfant). Mais le cœur du 
problème posé, c'est-à-dire l'intérêt d'associer les parents à la réeducation, reste inchangé. $Y$ avait-il d'ailleurs un problème, sinon dans la mise en scène qu'implique le mémoire?

«L'interaction humaine demeure complexe... » conclut l'étudiant. Le mémoire semble jover un rôle de renforcement de la solution proposée au problème dès le départ. II a mis en scène une démarche qui se veut répondre à des normes "scientifiques", mais il montre que finalement c'est dans la réflexion quotidienne (un savoir pratique, les données existentielles) que l'orthophoniste peut trouver les bonnes réponses pour ces enfants et familles démunis. Le problème reste posé de la même façon qu'au départ. Les travaux scientifiques présentés ne sont retenus que pour la mise en scène du problème et le questionnaire sert de masque vu comme nécessaire à cette mise en scène.

\section{LE MÉMOIRE COMME LIEU DE LA PROBLÉMATISATION}

Ces différentes analyses (qui ne prétendent à aucune généralisation) nous permettent de nous interroger sur l'outil mémoire comme lieu de problématisation.

\section{La mise en scène du problème}

Les artifices argumentaires et textuels produits par les auteurs des mémoires font revivre le cheminement intellectuel d'un processus de problématisation emblématique de l'exercice professionnel considéré, et qui en outre n'est pas toujours le problème réel qui hante l'étudiant. En effet, alors que l'on attend de ce dernier la manifestation d'un recul réflexif sur les tâches effectuées et sur sa propre démarche de professionnalisation, le problème qui se pose à lui est plutôt celui de la maîtrise des codes sociaux et des normes de jugement caractérisant le monde dans lequel évolvent ses évaluateurs (dans la formation et dans le contexte professionnel). L'exercice paraît donc doublement artificiel pour l'étudiant sommé de problématiser sa pratique et confronté au problème de la rédaction d'un bon mémoire professionnel, c'est-à-dire de créer un semblant symbolique suffisamment expressif de sa compétence.

\section{- Le mouvement et les procédés rhétoriques: artifices de mise en scène de la problématisation}

Dans les mémoires, l'expression initiale est celle d'une situation instable, non satisfaisante (souvent dans l'introduction ou dans la première partie) et l'énoncé d'objectifs et d'hypothèses de remédiation: il s'agit de permettre à un client une meilleure utilisation de fichiers informatiques, de permettre à des élèves de dépasser leurs difficultés en mathématiques, de permettre à des enfants de tirer un meilleur profit de leur rééducation orthophonique. Ensuite, l'étudiant recense des constats et tente d'analyser le problème. À ce moment, il fait appel à des savoirs hétérogènes (issus 
de sa propre pratique, diffusés dans la profession comme "savoirs ordinaires», ou encore savoirs scientifiques) qu'il agence de façon tatônnante et souvent cumulative. Dans certains mémoires (plutôt ceux des enseignants et des orthophonistes), on observe un retour réflexif sur les savoirs mobilisés (moi, la science, le monde) et leur mise en relation problématique. Mais dans aucun d'eux le problème n'est abordé dans l'ensemble de ses dimensions. Si les stagiaires sortent de ce que l'on peut appeler la pensée "plate», les articulations proposées, l'organisation en données et conditions, et même (en particulier pour l'orthophoniste) la délimitation du problème ne présentent pas les caractéristiques d'une problématisation. II s'agit plutôt, comme nous l'avons dit, d'une «pensée relationnelle en émergence» (Guigue, 1995).

Pourtant la dimension performative des conclusions amène des transformations (ou des propositions de transformation) de la pratique, des solutions à mettre en œuvre. Ces conclusions annoncent que le problème a été en partie clarifié: pour les ingénieurs, les solutions techniques proposées sont efficaces; pour l'enseignant le problème reste ouvert, il a retenu des conditions, en a exclu d'autres, mais un ensemble de possibles restent à explorer; pour l'orthophoniste au contraire, il semble que le problème soit résolu et la solution s'impose (même si le déploiement "scientifique» ne sert pas vraiment à prouver ce qui était avancé au départ).

\section{- Les spécificités des contextes professionnels}

Les postures, liées aux cultures professionnelles de ces milieux, amènent des façons différentes de poser les problèmes, et donc $d^{\prime}$ en (re) présenter une construction. Dans les représentations que s'en font les stagiaires, la légitimité professionnelle repose sur certaines données et la manière de problématiser met l'accent sur les modalités qui leur semblent attendues dans le contexte. Le «bon » ingénieur résout des problèmes techniques extérieurs à sa subjectivité; le "bon » orthophoniste articule des savoirs techniques et des savoirs humains, mais qu'il tente d'objectiver; le "bon» enseignant, selon une mise en scène épistémique, se remet en question dans sa réflexion et devient l'un des éléments du problème. On peut avancer que la formation des ingénieurs est encore marquée par une approche positiviste de la science: "le monde est comme cela et la science nous dit que..." semblent dire les stagiaires. Pour les orthophonistes le savoir existe et il suffit de le chercher, mais ce savoir n'est pas encore stabilisé (les théories sur le bégaiement sont multiples et il n'y a pas consensus). Il s'agit d'un métier entre la science/technique et l'humain. Pour l'enseignant, un "métier de l'humain » où les savoirs sont fragiles, la place du sujet et le rapport à soi comme sujet pensant est central, et l'étudiant se met en scène comme I'un des éléments du problème, peut-être influencé qu'il est par le paradigme du praticien réflexif.

Ces limitations intellectuelles peuvent apparaître comme des formes d'autocensure qui entravent la capacité à problématiser, dans la mesure où l'accès à la modalité 
problématique suppose sans doute des allers-retours dynamiques entre toutes les modalités du rapport au savoir: ontique pour la définition de la réalité observable, épistémique pour la distance réflexive au monde et à soi-même, pragmatique pour la mise en œuvre des hypothèses, axiologique pour l'évaluation conjointe de l'environnement et des hypothèses émises. Les mémoires étudiés sont loin d'y parvenir.

\section{CONCLUSION \\ Les limites de la forme "mémoire " comme lieu de problématisation}

Compte tenu des limites institutionnelles et formelles (Gonnin-Bolo, Benoit, 2004) et au vu des différentes productions de mémoires, qui illustrent la difficulté de la mise en scène rhétorique, il paraît assez vain d'attendre de la part de novices une véritable problématisation en actes. L'étudiant, plongé qu'il est dans un contexte professionnel, tout en restant attaché par le pied au monde de la formation, se doit d'identifier le problématique et de le circonscrire à ce qui sera pertinent pour illustrer sa compétence à problématiser. Son effort est tendu vers la conformation de ses travaux aux normes académiques sanctionnant l'obtention de son diplôme. Par cette mimesis (au sens aristotélicien de représentation intellectualisée du monde sensible) $d^{\prime}$ apprentissage il oriente la mise en scène du mémoire en fonction des injonctions des formateurs, et de ce qu'il sait ou croit être judicieux pour lui.

Mais la mise en scène ne parvient que difficilement à faire exister un processus de problématisation: on en reste alors au simple récit des tâches réalisées, à la reproduction d'un ensemble de savoirs savants extraits des cours reçus ou des livres lus, à la seule description de pratiques professionnelles et des techniques qu'elles mettent en œuvre. Pour l'étudiant, le mémoire comme exercice de mise en scène d'une compétence à problématiser se présente avant tout comme une forme $d$ 'injonction paradoxale: peut-on en effet forcer un étudiant à problématiser une question qu'il ne se pose pas encore, alors que ses problèmes existentiels sont tout autres?

Pour éviter ce malentendu récurrent, il paraît nécessaire de repenser, dans l'exercice du mémoire, l'apprentissage de la problématisation. Le mythe d'une problématisation "juste», "générale » et "finie » ne doit-il pas en effet être dépassé pour penser à la fois des degrés et des contextes de problématisation? En outre, ne faut-il pas considérer qu'il existe des classes de problèmes (techniques, scientifiques, humains...) et des contextes sociaux qui les condamnent à être posés de certaines façons? La problématisation apparaîtrait alors comme un processus qui déplace les questions, les articule différemment. Dans cette réflexion, c'est notre pensée qui risque de "boucler», en ce sens que c'est notre capacité à problématiser la problématisation qui se trouve en questions! 


\section{BIBLIOGRAPHIE}

FABRE M. (1999). - Situations-problèmes et savoir scolaire, Paris, PUF.

GOMEZ F. (2001). - Le mémoire professionnel, objet de recherche, outil de formation? Paris, Bruxelles, De Boeck Université.

GUIGUE-DURNING M. (1995). - Les mémoires en formation, Paris, L'Harmattan.

GONNIN-BOLO A. (2002). - «Le mémoire professionnel en IUFM, "traduction" des savoirs, "médiation" des formateurs", in Recherche et Formation, n 40, Paris, INRP.

GONNIN-BOLO A., BENOIT J.-P. (dir.) (2004). - Dix ans de mémoires professionnels en IUFM, Bilan de recherche et questions vives, Paris, INRP.

LEMAITRE D. (2003). - La formation humaine des ingénieurs, Paris, PUF.

POTIIER B. (1992). - Sémantique générale, Paris, PUF. 